\title{
Highlighting type A RRs as potential regulators of the dkHK1 multi-step phosphorelay pathway in Populus
}

\author{
F. Chefdor ${ }^{\mathrm{a}}$, F. Héricourt ${ }^{\mathrm{a}}$, K. Koudounas ${ }^{\mathrm{b}}$, I. Carqueijeiro ${ }^{\mathrm{b}}$, V. Courdavault ${ }^{\mathrm{b}}$, F. Mascagni ${ }^{\mathrm{c}}$, \\ L. Bertheau ${ }^{\text {a }}$, M. Larcher ${ }^{\text {a }}$, C. Depierreux ${ }^{\text {a }}$, F. Lamblin ${ }^{\text {a }}$, M.L. Racchi ${ }^{\mathrm{d}}$, S. Carpin ${ }^{\mathrm{a}, *}$ \\ ${ }^{\mathrm{a}}$ LBLGC, Université d'Orléans, INRA, USC1328, 45067, Orléans Cedex 2, France \\ ${ }^{\mathrm{b}}$ Biomolécules et Biotechnologies Végétales (BBV), EA 2106, Université François Rabelais de Tours, 31 avenue Monge, 37200 Tours, France \\ c Università di Pisa, Dipartimento di Scienze Agrarie, Alimentari e Agro-ambientali, Via del Borghetto 80, 56124 Pisa, Italy \\ d Scienze delle Produzioni Agroalimentari e dell'Ambiente, sezione di Genetica agraria, via Maragliano, 7550144 Firenze, Italy
}

\section{A R T I C L E I N F O}

\section{Keywords:}

Multistep phosphorelay

Type A response regulators

Populus

\begin{abstract}
A B S T R A C T
In previous studies, we highlighted a multistep phosphorelay (MSP) system in poplars composed of two hybridtype Histidine aspartate Kinases, dkHK1a and dkHK1b, which interact with three Histidine Phosphotransfer proteins, dkHPt2, 7, and 9, which in turn interact with six type B Response Regulators. These interactions correspond to the dkHK1a-b/dkHPts/dkRRBs MSP. This MSP is putatively involved in an osmosensing pathway, as dkHK1a-b are orthologous to the Arabidopsis osmosensor AHK1, and able to complement a mutant yeast deleted for its osmosensors. Since type A RRs have been characterized as negative regulators in cytokinin MSP signaling due to their interaction with HPt proteins, we decided in this study to characterize poplar type A RRs and their implication in the MSP. For a global view of this MSP, we isolated 10 poplar type A RR cDNAs, and determined their subcellular localization to check the in silico prediction experimentally. For most of them, the in planta subcellular localization was as predicted, except for three RRAs, for which this experimental approach gave a more precise localization. Interaction studies using yeast two-hybrid and in planta BiFC assays, together with transcript expression analysis in poplar organs led to eight dkRRAs being singled out as partners which could interfere the dkHK1a-b/dkHPts/dkRRBs MSP identified in previous studies. Consequently, the results obtained in this study now provide an exhaustive view of dkHK1a-b partners belonging to a poplar MSP.
\end{abstract}

\section{Introduction}

Living organisms are exposed to fluctuating environmental conditions, and in the context of global climatic change, those with efficient perception mechanisms will be able to adapt more efficiently. To sense and respond to environmental stimuli, prokaryotic organisms, such as bacteria, employ a well characterized signaling pathway, the canonical two component system (TCS). This system is specific to the stimuli and is comprised of a sensor protein corresponding to a Histidine Kinase and a Response Regulator (RR) [1]. A more elaborate system is also found in prokaryotic and eukaryotic organisms, which involves a His-Asp-HisAsp phosphorelay called a multistep phosphorelay (MSP) system. The system is comprised of sensors, RRs, and a histidine phosphotransfer protein (HPt), which shuttles between them [2]. In plants, the MSP comprises a receptor, a hybrid-type histidine aspartate kinase (HK), $\mathrm{HPt}$ proteins, and four types of RR: type A, type B, type C, and pseudo RRs. These signaling pathways are known to mediate plant responses to light, abiotic stresses, and phytohormones such as cytokinin (CK) and ethylene, which regulate growth and plant development [3-7].

The CK pathway is the most studied and characterized MSP in plants. In Arabidopsis, CK receptors AHK2, AHK3 and AHK4 activated by $\mathrm{CK}$ binding can autophosphorylate and trigger a phosphorelay involving five HPt proteins (AHP1-5), and 11 type B RRs (AtRRB1-2, AtRRB10-14, AtRRB18-21), which once activated by phosphorylation can activate the transcription of target genes such as type A RR genes [8-12]. These type A RR genes are primary response genes, and once activated by phosphate transfer via AHK and AHP proteins, act as negative regulators of CK signaling [9,10,13-15]. Type A RRs are consequently in competition with type B RRs for the phosphate transfer by AHP proteins in CK signaling and could also function via phosphorylation-dependent protein-protein interactions with type B RRs, or as yet non-identified regulators [14].

Type A RRs are also regulated by abiotic stresses such as drought, salinity, dehydration and cold [16-18]. For example, the expression of

\footnotetext{
* Corresponding author.

E-mail address: sabine.carpin@univ-orleans.fr (S. Carpin).
} 
the OSRRA1-2-5-6-7 and 9 from Oryza sativa are induced by salt, dehydration and low temperature treatments [16,17]. In Arabidopsis thaliana, AtRRA7 is cold inducible [18], and also acts as a transcriptional repressor for a variety of early CK-regulated genes such as genes encoding transcription factors, or signal transmitters [19]. Other results suggest that AtRRA3-4-5 and 6 may function as positive regulators whereas AtRRA8 and 9 function as negative regulators under osmotic stress [20]. In Glycine max, there are six dehydration-repressed type A RR genes (GmRRA07-08-09 and GmRRA11-12-13) which encode AtRRA8 and AtRRA9 homologues respectively, providing evidence that these GmRRAs function in stress response and may act as negative regulators in a similar fashion to their orthologues AtRRA8 and AtRRA9 [21]. Similarly, GmRRA01 and GmRRA02 genes, AtRRA4 and AtRRA6 homologues respectively, are up-regulated in response to drought, suggesting that they may function as positive regulators in this stress response [21]. This regulation by abiotic stresses has led to the proposition that type A RRs may also form a complex network that is predominantly responsible for integration, fine-tuning and cross-talk of many plant signaling pathways [22]. To date, target genes of activated type A RRs which negatively regulate the CK pathway remain to be identified.

Less data is available for tree plant models. In Malus domestica, 19 type A RRs were identified by in silico analysis and a number of them are up-regulated by CK treatments [23]. In Pinus pinea, a type A RR gene PipiRRA1 was cloned and shown to be up-regulated in cotyledons after CK exposure, suggesting that it could play a crucial role in adventitious meristem formation [24]. Moreover, the PipiRRA1 homologous gene PipsRRA1, cloned in Pinus pinaster, also seems to be involved in meristem formation and may play a role in adventitious shoot meristem formation and somatic embryo development [25]. In Populus trichocarpa, 11 type A RRs, PtRRA1 to PtRRA11, were identified in silico, whereas only four were detected in Prunus persica [26,27]. Some of them are up-regulated in detached mature leaves after $1 \mathrm{~h}$ of CK treatment in Populus tremula $\times$ Populus alba [26]. Regarding type A RRs in tree models, some studies have been conducted, although none of them investigate the role of these proteins in MSP regulation.

In previous works, we identified poplar HK, dkHK1a and dkHK1b $[28,29]$. Both dkHK1s can functionally complement a Saccharomyces cerevisiae deletion mutant for its two osmosensors $\operatorname{Sin} 1$ and Sho1, which demonstrates their kinase and osmosensor functions in yeast. Protein interaction studies alongside transcript co-expression analysis in planta have been carried out to determine dkHK1 partners among the 10 poplar HPt proteins identified. Hence, three HPt partners have been retained $[29,30]$. A similar study led to the identification of six type B RRs, which could participate in a poplar MSP [31-33]. Taken together, these studies highlight a network for dkHK1a-b/dkHPt2-7-9/dkRRB12$13,16,18-19$ MSP, which could potentially be involved in drought stress response in poplars. As type A RRs are negative regulators of CK MSP and participate in the regulation of CK signaling, the role of these proteins in other MSP pathways remains to be elucidated.

To complete the dkHK1 MSP network previously identified, we decided to identify type A RRs in poplars and to study their putative implication in this signaling pathway, as potential regulators of this MSP. We managed to isolate 10 cDNAs encoding type A RRs in the poplar 'Dorskamp'. Then we identified the subcellular localization, and studied the interactions with the three dkHPts, the preferential interacting partners for dkHK1, by performing two-hybrid assays in yeast. Some interactions were validated by BiFC assay in plant cells, and the relevance of these interactions has been strengthened by co-expression analysis of transcripts of all the studied proteins in poplar organs (roots, stems, petioles and leaf blades). Taken together, these results define a protein network linked to dkHK1 in poplar and highlight that at least eight dkRRAs may participate in the MSP dkHK1a-b/dkHPts/dkRRBs as type B RR competitors through their interactions with HPt proteins.

\section{Materials and methods}

\subsection{Isolation of type A RR CDSs and phylogeny analysis}

We used the references of genes from Populus trichocarpa type A RRs (PtRRA1 to PtRRA11) [26] to search for their nucleotidic sequences in JGI Populus trichocarpa (v1.1). We also designed a specific primer pair corresponding to each RR, in order to isolate their coding sequence (CDS) from the poplar clone 'Dorskamp' (Populus deltoides (Bartr.) Marsh x P. nigra L.). Apart from for $d k R R A 11$, all PCRs were performed using a root cDNA library constructed using the Marathon cDNA Amplification Kit (Clontech) and Taq Advantage polymerase (Clontech), with primers at a final concentration of $0.2 \mu \mathrm{M}$. PCR products were cloned into pGEM-T easy vector (Promega), and sequenced and compared with P. trichocarpa type A RR sequences using ClustalW [34]. PCR was performed under the same conditions for $d k R R A 11$, but using cDNA libraries from stressed leaves [31], and a nested PCR (Results Section 4).

Deduced amino acid sequences of dkRRAs were aligned with those from Arabidopsis thaliana using Clustal Omega (https://www.ebi.ac.uk/ Tools/msa/clustalo/). The alignment was represented with a phylogram constructed using the neighbour-joining method in the phylogenetic software MEGA (v 6.06) (Pennsylvania State University, State College, PA, USA).

\subsection{Yeast two-hybrid assays}

The yeast two-hybrid assays were performed using a LexA DNAbinding domain encoding bait vector (pBTM116 referred to as pLex), and a Gal4 activation domain encoding prey vector (pGADT7, Clontech). RRA CDSs were cloned into the PGAD vector as EcoRI-XhoI fragments for dkRRA1 to dkRRA10, and XmaI-XhoI for dkRRA11; dkHPt CDSs were cloned into the pLex vector as previously described [29]. The yeast strain L40 $\Delta$ (MATa ade2-101 his3-200 leu2-3,112 trp1-901 ura3-52 LYS2::(lexA op)x4-HIS3 URA3:: (lexA op)x8-lacZ gal4A) was used for co-transformations according to the lithium acetate method from [35]. Co-transformed yeasts were selected onto leucine-trytophan lacking medium (-LW) for 4 days at $30^{\circ} \mathrm{C}$. For each interaction, overnight cell cultures with an Optical Density at $600 \mathrm{~nm}\left(\mathrm{OD}_{600}\right)$ of 0.5 for three dilutions $(1: 10,1: 100$ and 1:1000) were prepared. Five microliters of each cell suspension were dropped onto control medium -LW (-Leu, -Trp) and interaction selective medium -LWH (-Leu, -Trp, -His). Due to autoactivation of dkHPts, 3-amino-1, 2, 4-triazole (3 A T) was supplemented to -LWH medium at either 20 (dkHPt7 and 9) or $60 \mathrm{mM}$ (dkHPt2) according to [31]. Yeast cells grew for two or four days at $30^{\circ} \mathrm{C}$ for all interactions which were tested using two different reporter genes, HIS3 and LacZ (data not shown). All interactions were tested at least twice with 8 positive yeast clones.

\subsection{BiFC assays}

BiFC assays were conducted using the PSPYCE(MR) [36] and pSPYNE173 plasmids [37], which allow the expression of a protein fused to the $\mathrm{C}$ - or N-terminal of the split-yellow fluorescent protein (YFP) fragments, respectively. The CDSs of dkRRA8 and dkRRA10 were cloned into the SpeI site in frame with the C-terminal fragment of YFP. DkHPt2, dkHPt7 and dkHPt9 CDSs were cloned into the SpeI site in frame with the N-terminal fragment of YFP [30]. Transient transformation of Catharanthus roseus cells by particle bombardment and YFP imaging were performed according to [38] with adaptation for BiFC assays [37] and negative control used is described in [39].

\subsection{DkRRA transcript detection by RT-PCR}

This study was performed using the poplar clone 'Dorskamp'. Roots, stems, petioles and leaf blades of one month-old hydroponically grown 
rooted cuttings [40] were harvested and frozen. RNA extractions were carried out using the NucleoSpin RNA Plant mini kit (Macherey-Nagel). One $\mu \mathrm{g}$ of total RNA was reverse transcribed using M-MuLV Reverse Transcriptase RNase H- (Finnzyme), according to the manufacturer's procedure, and used as a template for PCR amplifications. Thirty or forty PCR cycles were performed to detect $d k R R A$ transcripts, and clathrin was used as an expression control gene. The amplified fragments were separated by $1.2 \%$ agarose gel electrophoresis, stained with ethidium bromide, and analyzed under UV light. All PCRs were performed in triplicate at least, and three independent biological replicates were performed.

\subsection{DkRRA localization by GFP-fused protein expression}

To express RRA-GFP fusion proteins, the CDSs of RRAs were amplified by PCR using specific primers extended by SpeI restriction sites at both extremities. The amplified CDSs were subsequently cloned using the pGEM-T Easy Vector (Promega) and checked by sequencing. After SpeI digestion, CDSs were cloned into the SpeI restriction site of pSCAcassette GFP [38], upstream to and in frame with the CDS of GFP. Transient transformation of $C$. roseus cells by particle bombardment and GFP imaging were performed using the nucleocytoplasmic CFP (pSCAcassette CFP-GUS) and nuclear mcherry (pSCA-cassette mcherry-GUSNLS) markers [37]. These two plasmids have been created by substituting the YFP coding sequence of the pSCA-cassette YFP-GUS plasmid (described in [38]) by the CFP or mcherry coding sequence followed by the addition of a bipartite nuclear localization sequence (NLS) through primers annealing at the $5^{\prime}$ end of the mcherry GUS coding sequence [37].

\section{Results}

\subsection{Isolation of 10 type A RRs in the poplar clone 'Dorskamp'}

Using sequence information from [26] and the JGI Populus trichocarpa (v1.1) database, we isolated $10 \mathrm{CDSs}$ which encode type A RRs in the poplar clone 'Dorskamp': $d k R R A 1$ to $d k R R A 11$. In our plant materials (root or leaves) and PCR conditions we were unable to isolate cDNA encoding for dkRRA9. Among the 10 CDSs, identities ranged from 51 to $89 \%$. All the deduced amino acid sequences of isolated $d k R R A$ CDSs share the DDK conserved residues characteristic of the receiver domain (RD) of RRs (type A and type B), belonging to the MSP. As already observed by [26], some $d k R R A$ genes are duplicated and constitute sister pairs (Fig. 1). The C-terminal end of the dkRRA1/2 pair is characterized by a serine and proline rich zone (Fig. 2) as described for the Arabidopsis homologues, AtRRA3 and AtRRA4 [13]. DkRRA10, encoded by an unduplicated gene, is characterized by a charged serine and a proline rich zone, resembling AtRRA7 and AtRRA15, as its charged C-terminal end is also enriched by serine residues, although with threonine instead of proline residues. As observed in the unrooted tree (Fig. 1), five dkRRAs, dkRRA3-7, group together but present different characteristics. DkRRA3-5 are characterized by a charged Cterminal end as observed for AtRRA8 and 9, whereas dkRRA6 and 7 are characterized by a charged proline and glutamine rich C-terminal end without corresponding type A RRs in Arabidopsis (Fig. 2). A last group is composed of dkRRA 8 and dkRRA11 which are not characterized by a C-terminal end like for the pairs AtRRA5/6 and AtRRA16/17.

\subsection{DkRRA localization}

DkRRA subcellular localizations were determined by transient expression of GFP-fused dkRRAs in C. roseus cells. GFP and a nuclear mCherry marker were used as nucleocytoplasmic and nuclear markers respectively (Fig. 3A1, A2). All dkRRA proteins fused with GFP at their C-terminal ends displayed a strict nuclear localization (Fig. 3B1, D1-H1, $\mathrm{J} 1, \mathrm{~K} 1$ ) that was confirmed by the co-localization of the GFP fluorescent

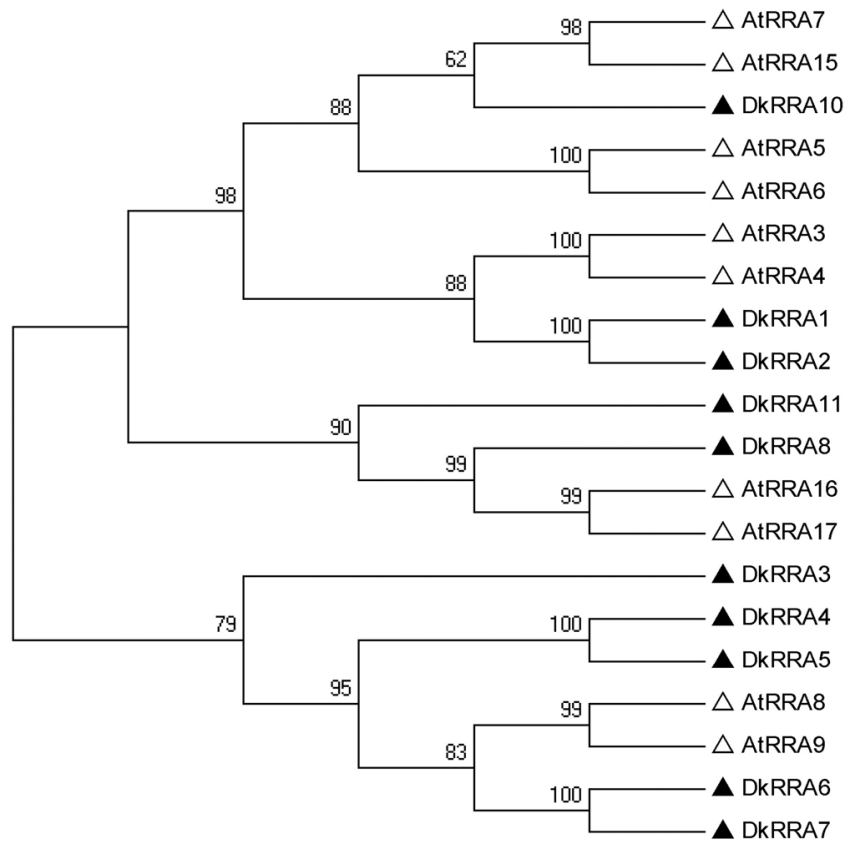

Fig. 1. Unrooted relationship tree of RRAs from Arabidopsis thaliana and Populus.

The full-length protein sequences of poplar type A RRs deduced from CDS sequences were aligned with those from Arabidopsis type A RRs using Clustal Omega, and the alignment was represented by a phylogram constructed with the neighbour-joining method in the phylogenetic software MEGA ( $\mathrm{v}$ 6.06). Numbers indicate bootstrap support (1000 replicates). $\Delta$ : AtRRAs, $\boldsymbol{\Delta}$ : dkRRAs.

signal with the signal of the nuclear mCherry marker (Fig. 3B3, D3-H3, J3, K3), except dkRRA2 and dkRRA8. Indeed, both dkRRA2- and dkRRA8-GFP fusion proteins showed an additional diffuse pattern of fluorescence, characteristic of a cytosolic localization (Fig. 3C1, I1). Using the nuclear mCherry marker (Fig. 3C2, I2), a nucleocytoplasmic localization was observed for both proteins, as they perfectly merged in the nucleus (Fig. 3C3, I3). Therefore, many of the dkRRA proteins showed a strict subcellular localization in the nucleus, except for dkRRA2 and dkRRA8 which exhibited an additional cytoplasmic localization.

\subsection{DkRRAs interact with dkHPt2, dkHPt7 and dkHPt9}

To determine whether dkRRAs could compete the interaction between dkHPt2-7-9 and dkRRB12-13, 16, 18-19, we performed twohybrid assays in yeast with all isolated type A RRs and the three dkHPt partners of dkHK1a-b, dkHPt2, 7 and 9. This study revealed reporter gene activation for all interactions tested except for dkRRA8, which only interacts with dkHPt7 (Fig. 4). Yeast expressing both dkRRA8 and dkHPt 2 or 9 showed a similar growth pattern with the negative control (Fig. 4A). For the dkRRA1/dkRRA2 pair, a different behavior was observed, as yeast expressing dkRRA2 needed two extra days before cell growth was observed (Fig. 4B). This delay probably reflects a weaker interaction between dkRRA2 and the three dkHPts, compared to dkRRA1. In the same way, a differential behavior was observed for dkRRA4/dkRRA5 and dkRRA6/dkRRA7 pairs with weaker growth observed for yeast expressing dkRRA5, compared to dkRRA4 and dkRRA6 to dkRRA7. The lack of dkRRA9 prevented a study into dkRR9/dkRR11 pair interactions. These two-hybrid assays showed that dkRRAs can be classed into four different categories according to reporter gene activation. The first group comprised dkRRA2, which presented weak interaction with the three dkHPt proteins. A second group included dkRRA1, 3, 4 and 7, and presented more pronounced interactions. An intermediate group was composed of dkRRA5, 6, 10 and 11, and the 


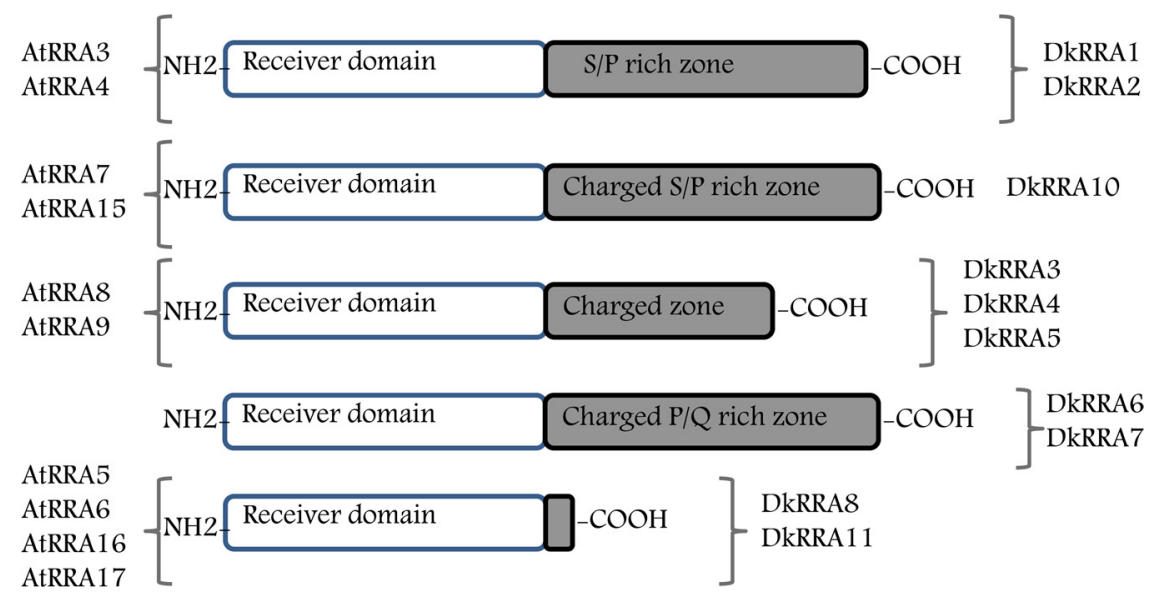

Fig. 2. Proteic characteristics of poplar type A RRs and correspondences with type A RRs from Arabidopsis thaliana.

last group was comprised of dkRRA8, which only interacted with dkHPt7.

\subsection{Validation of dkRRA8/dkHPt interaction by a BiFC approach}

To validate the lack of interaction observed in yeast two-hybrid assays between dkRRA 8 and dkHPt2 and 9, BiFC assays were conducted between dkRRA8 and the three dkHPt proteins in planta. DkHPt2, 7 and 9, dkRRA8, and dkRR10 CDSs were fused either to the N-terminal $\left(\mathrm{YFP}^{\mathrm{N}}\right)$ or C-terminal $\left(\mathrm{YFP}^{\mathrm{C}}\right)$ fragments of yellow fluorescent protein (YFP) at their C-terminal end, to produce $\mathrm{YFP}^{\mathrm{C}}$-dkHPt2/7/9 and dkRRA8/10-YFP ${ }^{\mathrm{N}}$. As observed in yeast two-hybrid tests, the BiFC approach substantiates the interaction between dkRRA 8 and dkHPt7 by observation of the YFP complex reconstitution (Fig. 5B1). Interactions between dkRRA 8 and dkHPt9 and dkHPt2 were observed using this second approach when co-expressing dkRRA8-YFP ${ }^{\mathrm{N}}$, and $\mathrm{YFP}^{\mathrm{C}}$-dkHPt2 (Fig. 5A1), and dkRRA8-YFP ${ }^{\mathrm{N}}$ and $\mathrm{YFP}^{\mathrm{C}}$-dkHPt9 (Fig. 5C1). As a positive control, we tested dkRRA10-YFP ${ }^{\mathrm{N}}$ with $\mathrm{YFP}^{\mathrm{C}}$-dkHPt2/7/9. As expected, the YFP complex reconstitution was observed for all interactions tested (Fig. 5D1, E1, F1). This signal using dkRRA10 merged perfectly with the CFP nuclear marker (Fig. 5D2, E2, F2) leading to the observation of a nuclear localization of the interaction due to the nuclear localization of dkRRA10 (Fig. 5D3, E3, F3). In contrast, BiFC complex reconstitution for dkRRA8-YFP ${ }^{\mathrm{N}}$ and $\mathrm{YFP}^{\mathrm{C}}$-dkHPt2/7/9 was observed both in nuclear and cytosolic compartments (Fig. 5A1, B1, C1). The localization in the nucleus was confirmed by the CFP nuclear marker (Fig. 5A2, B2, C2) and the merge observed (Fig. 5A3, B3, C3). This experiment led to the validation of the nuclear and cytoplasmic localization for the interactions of both the partners respectively. To confirm our results, negative controls were realized using CrTHAS1, a nuclear protein (Fig. 6A1, A2, A3). No interaction was observed either with $\mathrm{YFP}^{\mathrm{C}}$-dkHPt2/7/9 or with dkRRA8/10-YFP ${ }^{\mathrm{N}}$ (Fig. 6B1, C1, D1, E1, F1) respectively.

\subsection{DkRRA transcript expression analysis by RT-PCR}

To be physiologically pertinent in plants, all interactions observed using yeast two-hybrid or BiFC assays need to be validated by the observation of the concomitant co-expression of both partners in the same organs. To validate the relevance of observed interactions, the $d k R R A$ transcript expression pattern was studied by RT-PCR analysis with Clathrin as the reference gene (Fig. 7C). A constitutive expression was observed for six dkRRA transcripts (dkRRA2, 3, 5, 6, 7 and 10) in all studied organs (Fig. 7A) in 30 PCR cycles. To refine these results, we performed 10 more cycles and were able to detect two other RRs, dkRRA1 and dkRRA4 (Fig. 7B). These two RRs are less abundant than the others, as they were detected after 40 PCR cycles, and $d k R R A 1$ was not detected in leaf blades (Fig. 7B). Furthermore, a differential gene expression can be observed for both gene pairs, $d k R R A 1 / d k R R A 2$ and $d k R R A 4 / d k R R A 5$. In contrast, the gene pair $d k R R A 6 / d k R R A 7$ shows a similar expression pattern. Among the most expressed $d k R R A s(2,3,5$, 6, 7, and 10 Fig. 7A) dkRRA10 shows the weakest expression in roots, with expression being slightly stronger in leaf blades (Fig. 7B).

\section{Discussion}

In this study, we successfully isolated 10 cDNA encoding type A RRs from the poplar clone 'Dorskamp'. These RRAs correspond to dkRRA1 to $d k R R A 11$ (apart from $d k R R A 9$ ), among the 11 genes identified in JGI Populus trichocarpa (v1.1).

In Arabidopsis, all type A RR genes are duplicated [41,27], whereas in poplar three genes ( $d k R R A 3, d k R R A 8$ and $d k R R A 10)$ don't seem to be duplicated [26]. However, as observed in Arabidopsis, genes homologous to these three genes are duplicated in another tree species: Malus domestica [23]. Gene duplication is now well known to contribute to the evolution of novel functions. In plants, about $64.5 \%$ of genes are paralogs, ranging from $45.5 \%$ in Physcomitrella patens to $84.4 \%$ in $M$. domestica, and the longevity of duplicated genes may be influenced by various factors [42]. For example, duplicate loss could be observed for weakly expressed genes with uncomplex promoters [43]. A similar lack of pairwise genes is observed for rice RRAs, probably due to frequent gene loss events [44]. Thus, the hypothesis that these three genes in poplar have never been duplicated or undergone a duplicate loss during poplar evolution could be considered.

The dkRRA1/dkRRA2 pair, homologous to the AtRRA3/AtRRA4 pair, shares the same characteristics, i.e. a RD followed by a serine and proline rich domain in the C-terminal end. In Arabidopsis, AtRRA4, was shown to interact with phytochrome B (PHY-B) and found to be involved in a phase delay of the circadian rhythm $[45,46]$. Moreover, AtRRA4 plays a central role in the interaction between cytokinin signaling and light signal transduction. Phosphorylation of the conserved aspartate residue in the $\mathrm{RD}$ is important for AtRRA4 activity during photomorphogenesis [47], but this activity is also controlled by its protease-mediated degradation [48]. This degradation is mediated by DEG9 interaction with AtRRA4's C-terminal end, leading to AtRRA4 specific degradation, which is not observed for AtRRA3 due to differences in the C-terminal end [48]. A similar C-terminal extension, characterized by a serine/proline rich domain, was found in dkRRA1/ dkRRA2, which suggests a possible involvement in circadian rhythm regulation in poplar for these two RRAs.

In the phylogenetic tree, dkRRA3, the dkRRA4/dkRRA5 and dkRRA6/dkRRA7 pairs group together with AtRRA8/AtRRA9. However, they do not share similar characteristics in their C-terminal ends. DkRRA3 and dkRRA4/dkRRA5 have charged C-terminal ends 


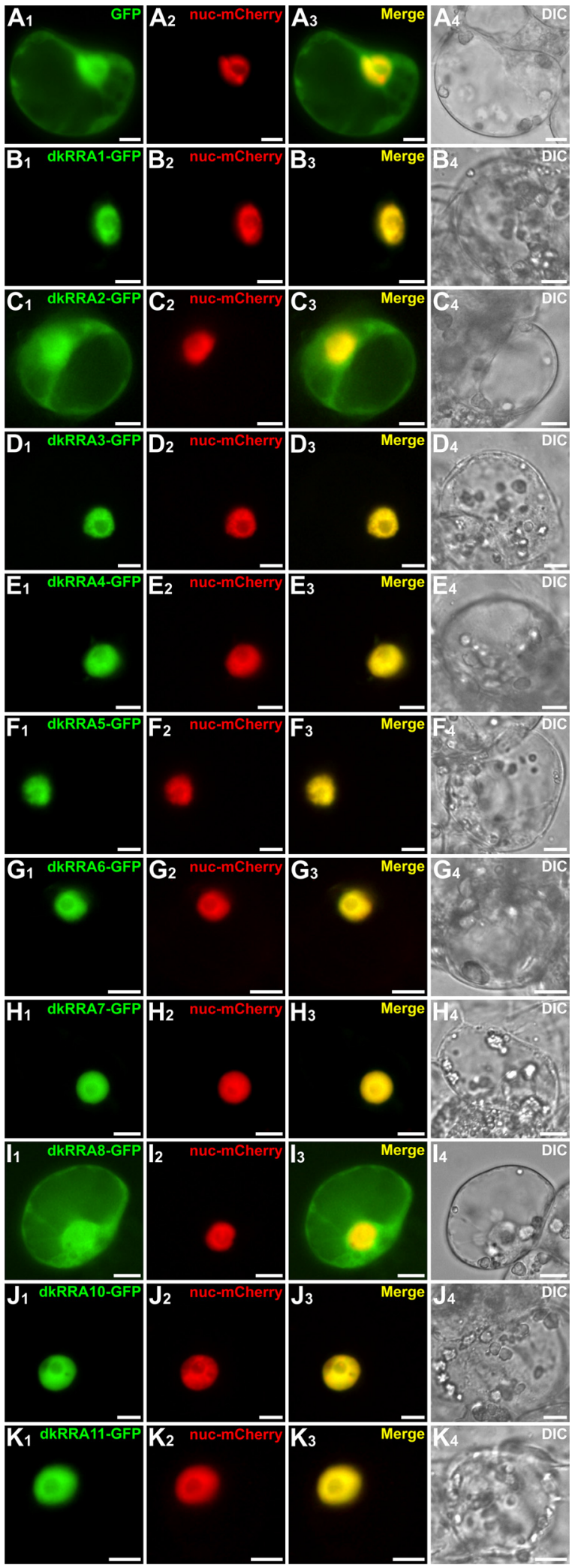

Fig. 3. Subcellular localization of poplar type A RRs.

C. roseus cells were transiently transformed with GFP (A1) and dkRRAs-GFP (B1-K1) expressing vectors in combination with a nuclear-mcherry marker (A2K2). Co-localization of the two fluorescence signals is shown in the merged image (A3-K3). The morphology was observed by differential interference contrast (DIC) microscopy (A4-K4). Scale bar $=10 \mu \mathrm{m}$. similar to AtRRA8 and AtRRA9, whereas the dkRRA6/dkRRA7 pair contains a short, charged proline/glutamine rich domain. No type A RRs from Arabidopsis have these domains. While type B RRs are characterized by a C-terminal end enriched in proline/glutamine residues involved in transactivation function, such domains are lacking in type A RRs [49,3], and this domain in dkRRA6 and dkRRA7 is not long enough to function as a transactivation domain. However, proline and glutamine residues are often associated with protein interactions [50], raising the question as to whether this domain could be involved in protein-protein interactions leading to specific function for these type A RRs as observed for AtRRA4's C-terminal end.

In the same way, dkRRA8 and dkRRA11 group with the AtRRA16/ AtRRA17 pair in the phylogenetic tree, as expected due to their common architecture, i.e. a short C-terminal end ( $<30$ amino acids). However, the AtRRA5/AtRRA6 pair does not group with these dkRRAs even though they share the same architecture. Moreover, dkRRA10, which is homologous to AtRRA5, shares common architecture, a serine and proline/threonine rich domain, with the AtRRA7/AtRRA15 pair. It should be mentioned that RR phylogenetic trees are commonly constructed using only the RD, and do not include the C-terminal end [41]. However, the C-terminal end of these proteins is clearly important for the protein function or regulation, as shown for AtRRA4 [48]. Thus, even though these proteins are phylogenetically distant, they could share common functional mechanisms thanks to their common architecture.

In our study, we failed to isolate one CDS, $d k R R A 9$, but we did succeed in isolating $d k R R A 11$ using a nested PCR on cDNAs from poplar drought stressed leaves. PtRRA9, homologous to $d k R R A 9$, was also undetectable in roots, young and mature leaves, nodes and internodes, phloem and xylem of two other poplar genotypes, Populus balsamifera ssp. trichocarpa genotype Nisqually 1 and Populus tremula $\times$ Populus alba INRA-clone 717-1-B4 [26]. In these two genotypes, PtRRA3, PtRRA9 and PtRRA11 were undetectable in these organs, although clearly expressed in catkins, from which only PtRRA9 was strictly specific. The PtRRA9 tissue specific expression in catkins linked to its involvement in sex determination could explain our unsuccessful attempt to isolate this RRA from our poplar material (roots, stems, petioles and leaf blades). It was shown that the sex-linked specific region in Populus trichocarpa contains 13 genes, with at least two candidate genes involved in sex determination: a methyltransferase, PtMET1 and a type A RR, PtRRA9, homologous to AtRRA17 [51]. In P. balsamifera, authors showed that PbRRA9 was more heavily methylated in males than in females and consequently probably less expressed in males, leading to the hypothesis that the PbRRA9 gene could be involved in poplar sex determination [52]. It was also demonstrated that male poplars adapt more efficiently during drought stress, as water deficiency inhibits growth, photosynthesis and ROS protection more strongly in females than in males [53]. Indeed, this difference was previously observed between two Populus $x$ euramericana clones, the male genotype 'Dorskamp' and the female genotype 'Luisa Avanzo' [54]. Growth and photosynthesis in female poplars were affected via a clear leaf area decrease during drought [55]. Furthermore, in Arabidopsis, leaf differentiation and consequently leaf area is controlled through AtRRA16 activation by the complex of the chromatin remodeler BRM and TCP4 CIN-TCP leading to CK decrease [56,57]. It seems that type A RRs could be involved in sex-specific drought response (AtRRA17) or leaf development and leaf area control (AtRRA16). In the poplar clone 'Dorskamp', dkRRA9 could be also involved in sex determination, and therefore indirectly in drought response. The fact that it was impossible to isolate this gene in vegetative tissue could argue in favor of this hypothesis. Moreover, the increase of dkRRA11 transcript levels in drought stressed leaves compared to the control in nested RT-PCR (data not shown, condition to isolate the corresponding CDS) raised the question about this RRA's involvement in leaf area control during drought. As neofunctionalization after whole genome duplication has been proposed for PtRRA9/PtRRA11 [51,52], 
A

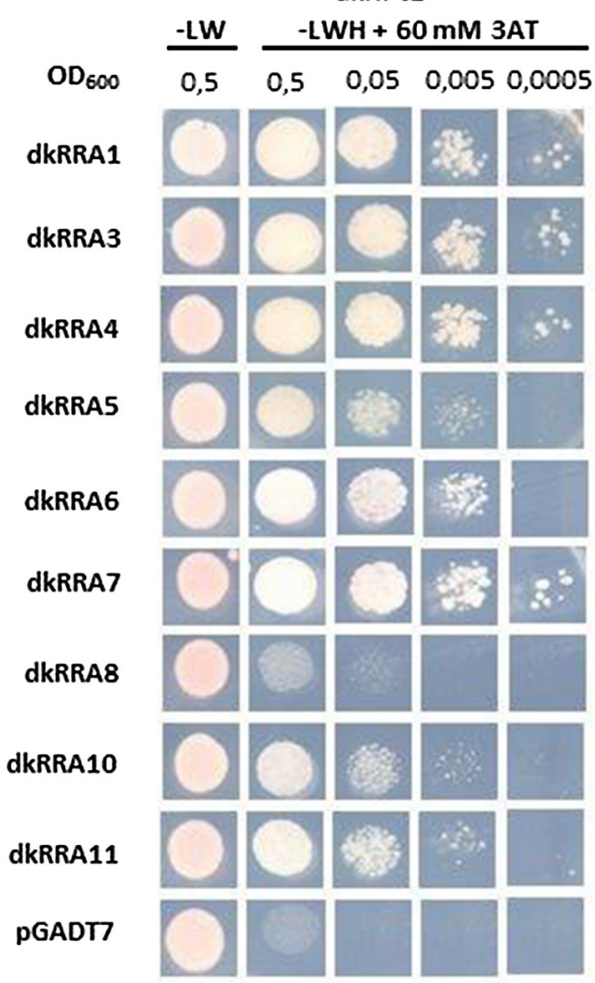

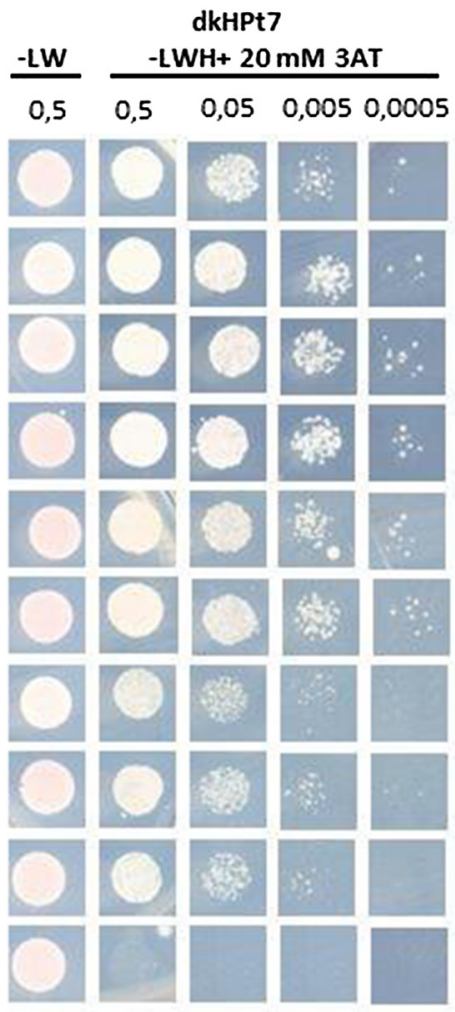

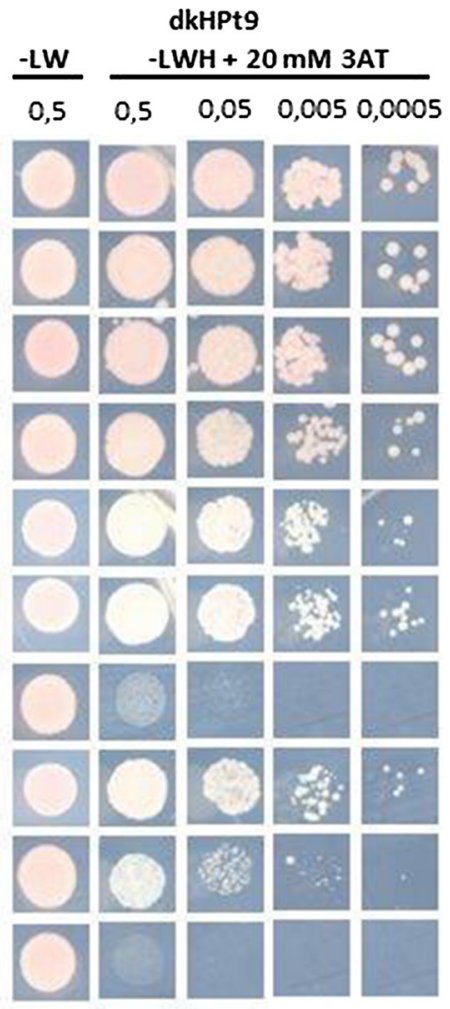

B

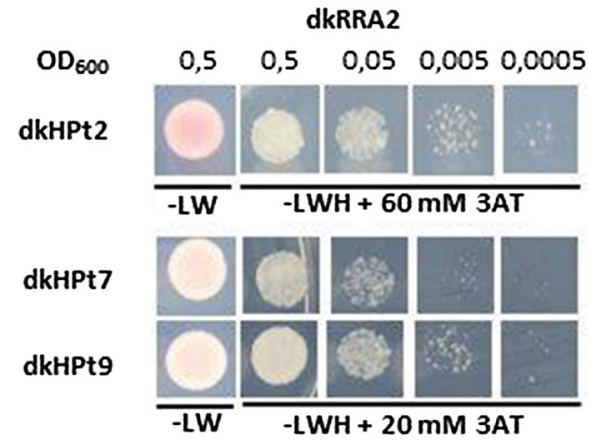

Fig. 4. Interaction between dkRRAs and dkHPt2-7-9 in the yeast two-hybrid system. Overnight cultures of yeast co-transformed with pLex-dkHPts and pGAD-dkRRAs were adjusted to an Optical Density at $600 \mathrm{~nm}(\mathrm{OD} 600)$ of 0.5 . This culture and three dilutions $\left(\mathrm{OD}_{600} 0,05\right.$ to 0,0005$)$ were spotted onto -LWH medium supplemented with $3 \mathrm{~A} \mathrm{~T}$ as indicated and grown for two (A) or four (B) days.

more studies are needed to determine the involvement of this RRA in poplar drought response.

Since only in silico prediction methods have been used to analyze poplar type A RR subcellular localization until now, we have done an exhaustive study to confirm or reject these predictions in planta. These assays showed a refined localization for dkRRA2, 4 and 5 compared to in silico results obtained by Ramirez-Carvajal et al. [26], hence pointing out the necessity to perform experimental validation. DkRRA1 is a nuclear protein, whereas AtRRA4, dkRRA2 and AtRRA3 are all nucleocytoplasmic proteins [58]. Because nuclear AtRRA4 is involved in mediating cross-talk between light and CK signaling through modulation of PHY B activity, and is involved in the circadian period, dkRRA1 could be involved in a similar process $[59,47]$. This observation, as well as common structural characteristics, could assume a similar cellular function of poplar proteins. DkRRA4, 5, 6 and 7 are nuclear proteins as is observed for their Arabidopsis homologues, AtRRA8 and AtRRA9 [58]. DkRRA8 is localized in the nucleus and cytoplasm, as is observed for AtRRA16, probably due to their same short C-terminal ends [60].

In the past, type A RRs were identified as primary response genes, and are known to be negative regulators in CK pathways by applying a negative feedback control in CK sensibility. This feedback control is probably due to their activation by phosphorylation, leading to a reduced degradation and an increased stability $[10,14,15,61]$. This activation is due to direct interaction with HPt proteins in the nucleus $[15,62,63]$.

In previous work, we identified a specific network of interactions composed of dkHK1a-b/dkHPt2-7-9/dkRRB12-13, 16, 18-19 [29-31,33]. To determine if type A RRs could interfere with this network, we decided to study potential interactions between all isolated type A RRs, and the three dkHPt protein partners of dkHK1. All type A dkRR proteins were able to interact with these dkHPt proteins. However, a surprising behavior was observed for type A RR pairs. Indeed, all pairs are composed of a strongly and a weakly interacting RRA. In Arabidopsis, AtRRA3/AtRRA4 and AtRRA8/AtRRA9 pairs showed similar interaction patterns for AHP5, and some differences were detected for AtRRA5/AtRRA6 and AtRRA7/AtRRA15 pairs [64]. These different binding properties of proteins making up each pair could reflect the redundancy already observed in other plant models. A different 

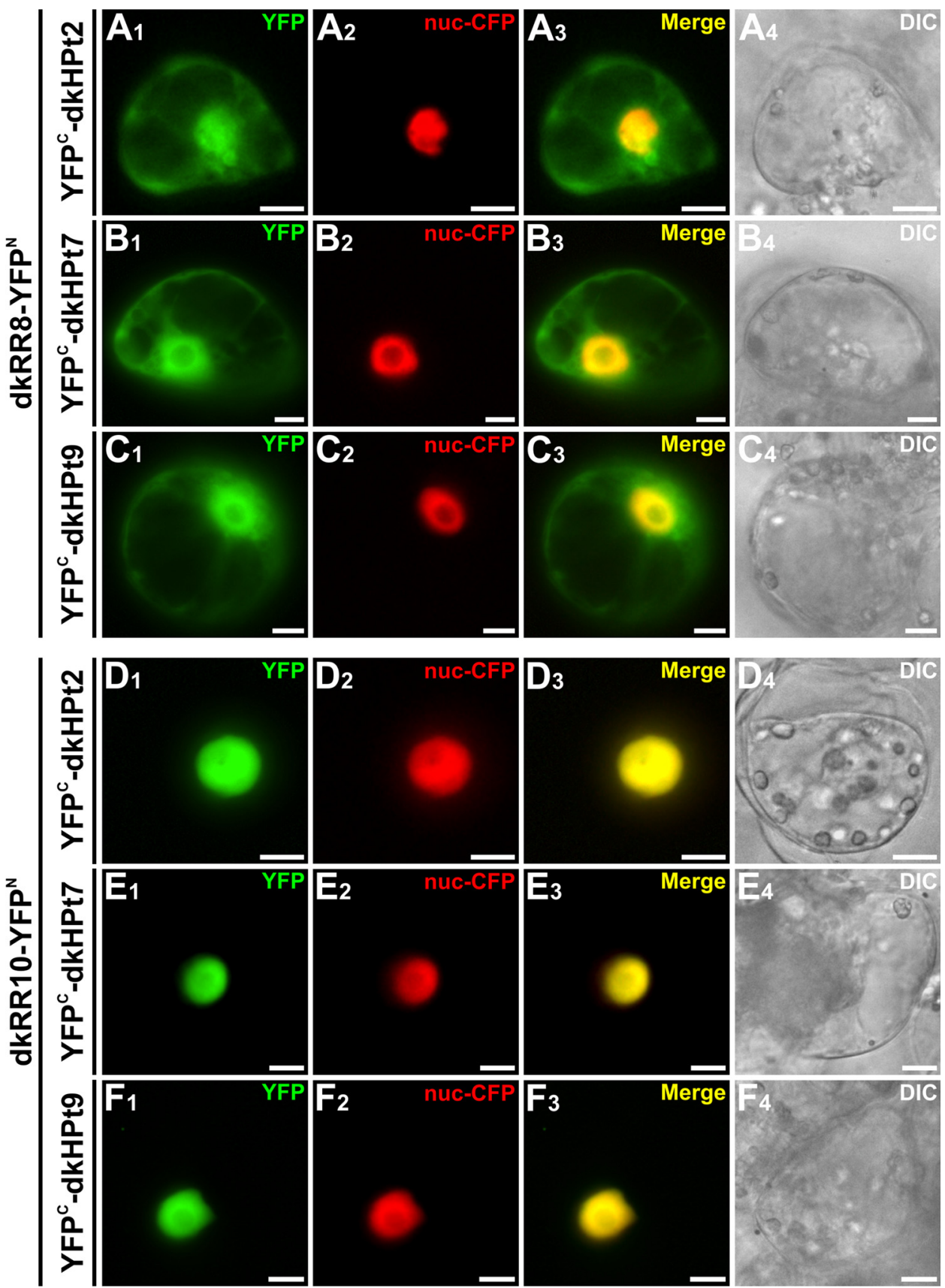

Fig. 5. Analysis of dkRRA8/dkHPt2-7-9 interactions in C. roseus cells using BiFC assays.

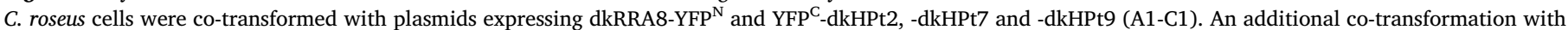

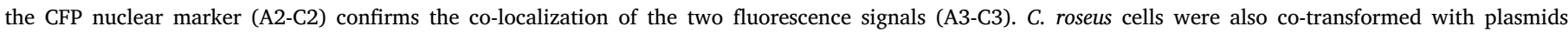

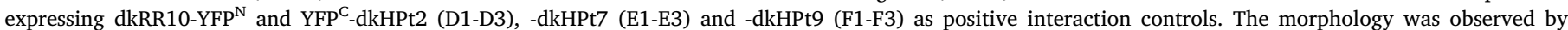
differential interference contrast (DIC) microscopy (A4-F4). Scale bar $=10 \mu \mathrm{m}$.

experimental approach is often necessary to confirm two-hybrid results [65,31]. The lack of interactions observed between dkRRA8 and dkHPt2/dkHPt9 was checked by BiFC and revealed interactions between dkRRA8 and these HPts. The strong sequence similitude between the dkHPt7 and dkHPt9 (96.7\%) pair was in favor of such results with dkHPt9. Moreover, the BiFC approach showed that the interactions of dkRRA8/dkHPts and dkRRA10/dkHPts exhibited a nucleocytoplasmic and a nuclear localization respectively.

The interactions observed in yeast two-hybrid or in planta BiFC assays are only biologically relevant if tested proteins are spatio-temporally co-expressed in the plant. As the expression of all proteins of the dkHK1a-b/dkHPt2-7-9/dkRRB12-13, 16, 18-19 network was studied in roots, stems, petioles and leaf blades [31,29], we decided to study the type A RR expression from the exact same biological material.

As previously discussed, the $d k R R A 9$ CDS was not isolated and consequently undetectable in our experiment. Surprisingly, constitutive expression for PtRRA9 transcripts was detected in leaves by RT-PCR in the genotype $P$. tremula $\times P$. alba, although three aberrant transcripts shorter than the expected one were found [26]. Consequently, in these poplar genotypes, Populus RRA9 is probably not expressed in leaves. $D k R R A 8$ and $d k R R A 11$ transcripts were also undetectable in our experiment, even though we managed to isolate them from the cDNA library due to its enrichment in mRNA. In $P$. trichocarpa and $P$. tremula $x$ $P$. alba, PtRRA11 transcripts were also undetectable in all vegetative 


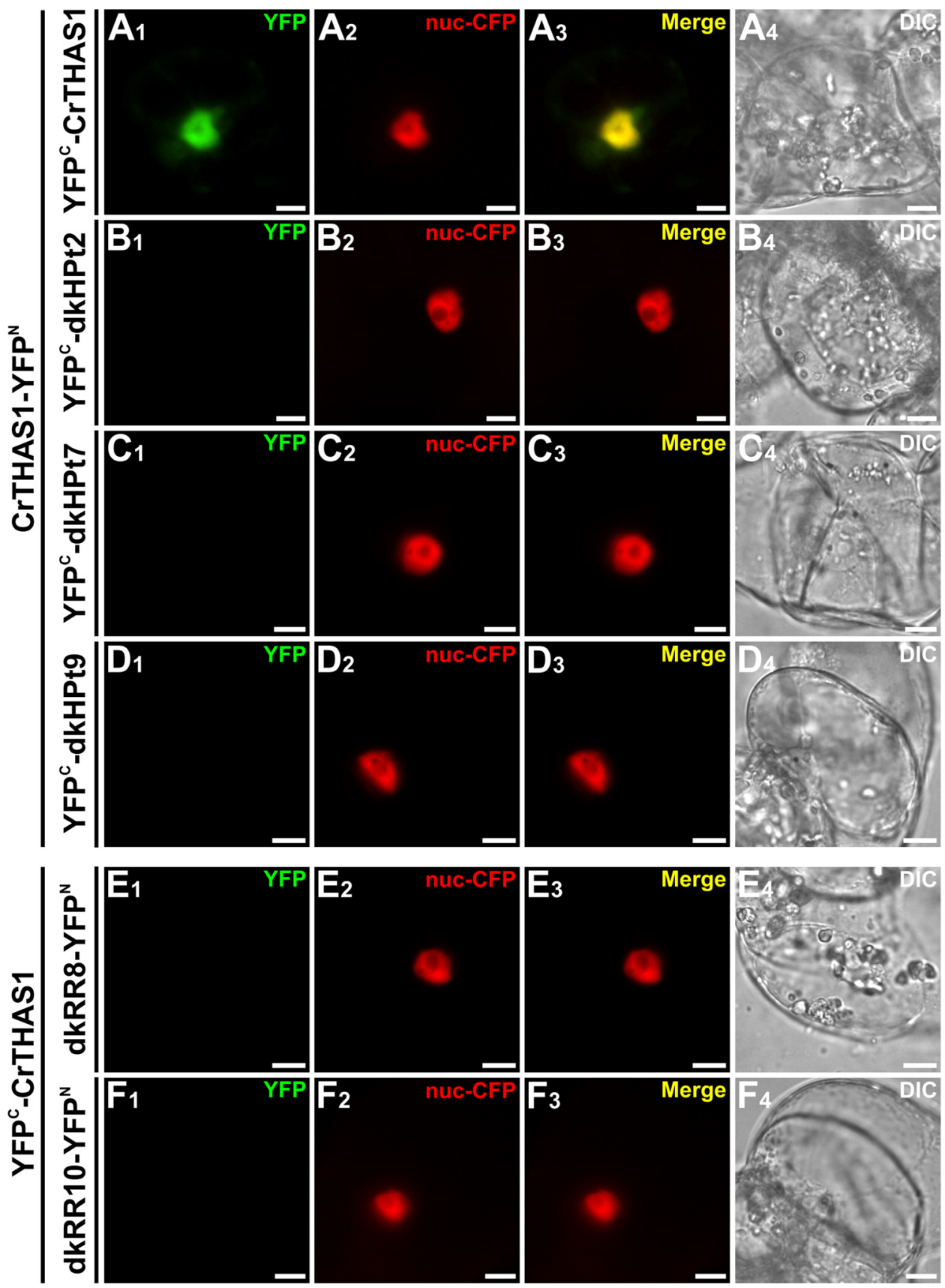

Fig. 6. Control of dkRRA8-10/dkHPt2-7-9 interaction specificity in C. roseus cells using BiFC assays. C. roseus cells were co-transformed with plasmids expressing YFP $^{\mathrm{C}}$-CrTHAS1 with dkRRA8 (E1) and dkRRA10YFP ${ }^{\mathrm{N}}$ (F1), and CrTHAS-YFP ${ }^{\mathrm{N}}$ with YFP $^{\mathrm{C}}$-dkHPt2 (B1), -dkHPt7 (C1) and -dkHPt9 (D1). An additional co-transformation with the CFP nuclear marker (A2-F2) specifies the nuclear localization. A positive control is observed by transformation with YFP ${ }^{\mathrm{C}}$-CrTHAS1 with CrTHAS$\mathrm{YFP}^{\mathrm{N}}$ (A1, B1, C1). The morphology was observed by differential interference contrast (DIC) microscopy (A4-F4). Scale bar $=10 \mu \mathrm{m}$.

organs studied (leaves and detached leaves, nodes, internodes and roots) but were slightly expressed in phloem, xylem and in catkins [26]. The same pattern of expression could most probably be expected in the 'Dorskamp' clone for dkRRA11. However, the isolation of $d k R R A 11$ from drought stressed leaves after a nested PCR supports the hypothesis that $d k R R A 11$ is slightly expressed in leaves and may be regulated by drought (data not shown). On the other hand, $d k R R A 8$ transcripts were detected in all vegetative tissues and precatkins in $P$. trichocarpa, but not in $P$. tremula $\times P$. alba leaves [26]. Another difference observed was that $d k R R A 3$ was expressed in the 'Dorskamp' clone, but not in $P$. trichocarpa or P. tremula $x$ P. alba [26]. Consequently, the lack of $d k R R A 8$, 9 and 11 gene expression in vegetative tissues studied led us to suppose that these three type A RRs could not interfere in the dkHPt2-7-9/
dkRRB12-13, 16, 18-19 network during the early drought response, since their mRNAs are not detected in control conditions. Surprisingly, dkRRA4-5-7 and 10 are similarly expressed in control leaves in these three genotypes (RRA9 and 11 are not detected and present a common response in these genotypes), whereas $d k R R A 1-2-3-6$, and 8 present various expression patterns, creating the hypothesis that there are genotype specific responses to stress (Table S1). Could these differences, observed between genotypes for the expression level of type A RRs under control conditions, explain the different adaptability of the genotypes to environmental constraints? A comparative study of genotype response during environmental constraints could help answer this question. In Populus $x$ canescens stems (corresponding to P. tremula $\times P$. alba) PtaRRA3 has been shown to be down regulated by drought [66]. 


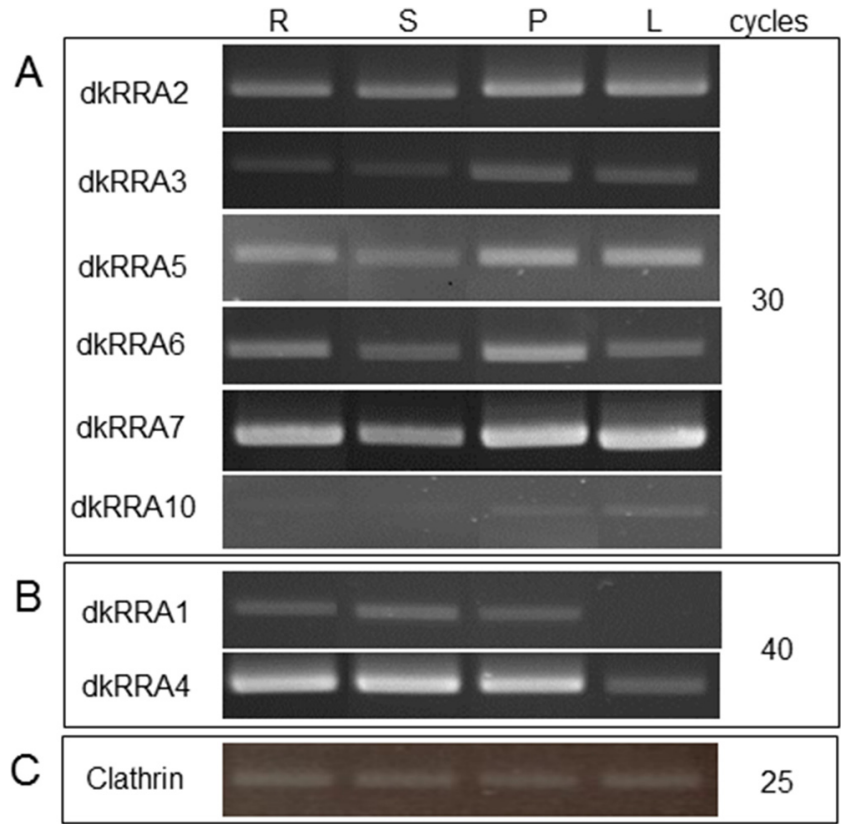

Fig. 7. Expression analysis of poplar type A RRs by RT-PCR.

RNAs isolated from roots (R), stems (S), petioles (P) and leaf blades (L) were reverse transcribed and used as templates for PCR amplification. PCR reactions were performed using $d k R R A$ specific primers under optimal conditions for each primer set (A: 30 cycles, B: 40 cycles). Expression profile of Clathrin, used as a housekeeping gene, was realized with 25 cycles of PCR amplification (C).
Due to this RRA3 regulation and the interaction between dkRRA3 and dkHPt2, 7 and 9, further studies should be conducted to investigate a possible involvement of this RRA as a regulatory protein during water stress. As shown in Arabidopsis seedlings, a slight increase in AtRRA5, 7 and 15 expression was observed during dehydration stress [67,68]. Since AtRRA5 is the homologue of PtRRA10, it could be interesting to investigate type A RR transcript regulation during drought in all organs by a semi-quantitative PCR approach during a more complete timecourse experiment.

In the present work, the aim of our analysis was to check if type A RRs could interfere with the dkHK1a-b/dkHPt2-7-9/dkRRB12-13, 16, 18-19 pathway previously characterized in poplar [28-33]. We successfully isolated 10 type A RRs, and defined their subcellular localizations experimentally, which weren't exactly as predicted by in silico tools. Moreover, we determined that since they were co-expressed with dkHPt proteins, eight of them could interfere in the MSP dkHK1a-b/ dkHPt2-7-9/dkRRB12-13, 16, 18-19 (Fig. 8). Amongst these eight interacting proteins, dkRRA1 and dkRRA10 could not interfere with MSP partners in leaves and roots respectively as they were either poorly expressed, or not expressed at all in these organs under our experimental conditions. Our results, together with literature data, show a variability of type A RR gene expression for three Populus genotypes under control conditions, leading to the possible conclusion that there is a genotypic variability for these proteins belonging to MSP. Consequently, variable poplar responses to unfavorable environmental conditions could be explained, at least in part, by this MSP genotypic variability which could lead to a better tolerance to stress. This hypothesis emphasizes the importance of a study into the genotypic variability of poplar MSP partners in this context of global climate change.

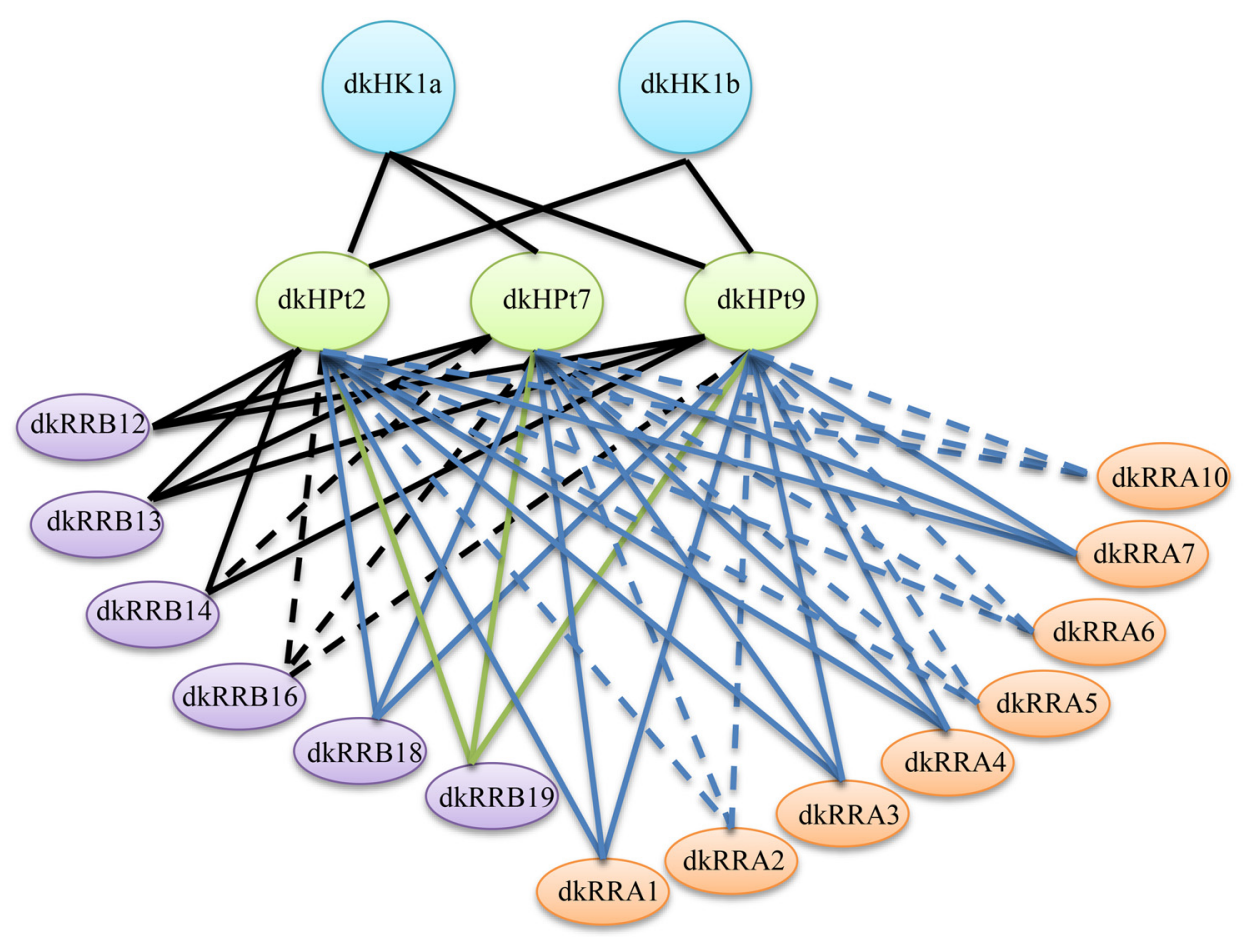

\footnotetext{
_ Interaction tested by two-hybrid, quantitative Oggal test and BiFC assay, strong affinity [29, 30, 31, 32]

- Interaction tested by two-hybrid, quantitative Ogal test and BiFC assay, weak affinity [31]

Interaction tested by two-hybrid, qualitative Ogal test, strong affinity [33, Chefdor et al.,]

$=-$ Interaction tested by two-hybrid, qualitative Ogal test, weak affinity and BiFC assay for dkRRA10 [33, Chefdor et al.,] Interaction tested by BiFC assay, strong affinity ${ }^{[31]}$
}

Fig. 8. Interaction network in dkHK1 multistep phosphorelay pathway. 


\section{Conflicts of interest}

The authors declare no conflict of interest.

The funding sponsors had no role in the design of the study; in the collection, analyses, or interpretation of data; in the writing of the manuscript, and in the decision to publish the results.

\section{Acknowledgements}

This work wassupported in LBLGC by grants from Conseil Régional du Centre-Val de Loire (SiSeP project no 2008-0033673) and in BBVEA2106 by funding the CatharSIS program and BioPROPHARM project - ARD2020 Biomédicaments). We would like to thank Dr Annette Nassuth and Dr Raymond Lee from Guelph University (Canada) and Ms Deki Fourcin from "Infinity English" for English reviewing.

\section{Appendix A. Supplementary data}

Supplementary material related to this article can be found, in the online version, at doi: https://doi.org/10.1016/j.plantsci.2018.09.010.

\section{References}

[1] L.A. Abriata, D. Albanedi, M. Dal Peraro, D. de Mendoza, Signal sensing and transduction by histidine kinases as unveiled through studies on a temperature sensor, Acc. Chem. Res. 50 (6) (2016) 1359-1366.

[2] A.F. Alvarez, C. Barba-Ostria, H. Silva-Jimenez, D. Georgellis, Organization and mode of action of two component system signaling circuits from the various kingdoms of life, Environ. Microbiol. 18 (2016) 3210-3226.

[3] I. Hwang, H.C. Chen, J. Sheen, Two-component signal transduction pathways in Arabidopsis, Plant Physiol. 129 (2002) 500-515.

[4] F. Schaller, P. Zerbe, S. Reinbothe, C. Reinbothe, E. Hofmann, S. Pollmann, The allene oxide cyclase family of Arabidopsis thaliana - localization and cyclization, FEBS J. 275 (2008) 2428-2441.

[5] T. Werner, T. Schmülling, Cytokinin action in plant development, Curr. Opin. Plant Biol. 12 (2009) 527-538.

[6] X. Shan, J. Yan, D. Xie, Comparison of phytohormone signaling mechanisms, Curr. Opi. Plant Biol. 15 (2012) 84-91.

[7] I. Hwang, J. Sheen, B. Müller, Cytokinin signaling networks, Annu. Rev. Plant Biol. 63 (2012) 353-380.

[8] A. Imamura, N. Hanaki, A. Nakamura, T. Suzuki, M. Taniguchi, T. Kiba, et al., Compilation and characterization of Arabiopsis thaliana response regulators implicated in his-asp phosphorelay signal transduction, Plant Cell Physiol. 40 (1999) 733-742.

[9] T. Kiba, H. Yamada, S. Sato, T. Kato, S. Tabata, T. Yamashino, T. Mizuno, The typeA response regulator, ARR15, acts as a negative regulator in the cytokinin-mediated signal transduction in Arabidopsis thaliana, Plant Cell Physiol. 44 (2003) 868-874.

[10] J.P.C. To, G. Haberer, F.J. Ferreira, J. Deruère, M.G. Mason, G.E. Schaller, et al., Type-A Arabidopsis response regulators are partially redundant negative regulators of cytokinin signaling, Plant Cell 16 (2004) 658-671.

[11] M.G. Mason, D.E. Mathews, D.A. Argyros, B.B. Maxwell, J.J. Kieber, J.M. Alonso, et al., Multiple type-B response regulators mediate cytokinin signal transduction in Arabidopsis, Plant Cell 17 (2005) 3007-3018.

[12] E. Ramireddy, W.G. Brenner, A. Pfeifer, A. Heyl, T. Schmuilling, In planta analysis of a cis-regulatory cytokinin response motif in arabidopsis and identification of a novel enhancer sequence, Plant Cell Physiol. 54 (2013) 1079-1092.

[13] I.B. D'Agostino, J. Deruère, J.J. Kieber, Characterisation of the response of the Arabidopsis response regulator gene family to cytokinin, Plant Physiol. 124 (2000) 1706-1717.

[14] J.P.C. To, J. Deruère, B.B. Maxwell, V.F. Morris, C.E. Hutchison, Ferreira, et al., Cytokinin regulates type-A Arabidopsis response regulator activity and protein stability via two-component phosphorelay, Plant Cell 19 (2007) 3901-3914.

[15] J.P.C. To, J.J. Kieber, Cytokinin signaling: two-components and more, Trends Plant Sci. 13 (2) (2008) 85-92.

[16] M. Jain, A.K. Tyagi, J.P. Khurana, Molecular characterization and differential expression of cytokinin-responsive type-A response regulators in rice (Oryza sativa), BMC Plant Biol. 6 (2006) 1-11.

[17] M. Jain, A.K. Tyagi, J.P. Khurana, Differential gene expression of rice two-component signaling elements during reproductive development and regulation by abiotic stress, Funct. Integr. Genomics 8 (2008) 175-180.

[18] J. Jeon, N.Y. Kim, S. Kim, N.Y. Kang, O. Novak, S.-J. Ku, et al., A subset of cytokinin two-component signaling system plays a role in cold temperature stress response in Arabidopsis, J. Biol. Chem. 285 (2010) 23371-23386.

[19] D.J. Lee, J.-Y. Park, S.-J. Ku, Y.-M. Ha, S. Kim, M.D. Kim, et al., Genome-wide expression profiling of Arabidopsis response regulator 7 (ARR7) overexpression in cytokinin response, Mol. Genet. Genomics 277 (2007) 115-137.

[20] D.J. Wohlbach, B.F. Quirino, M.R. Sussman, Analysis of the Arabidopsis histidine kinase ATHK1 reveals a connection between vegetative osmotic stress sensing and seed maturation, Plant Cell 20 (2008) 1101-1117.
[21] D.T. Le, R. Nishiyama, Y. Watanabe, K. Mochida, K. Yamaguchi-Shinozaki, K. Shinozaki, L.-S.P. Tran, Genome-wide expression profiling of soybean twocomponent system genes in soybean root and shoot tissues under dehydration stress, DNA Res. 18 (2011) 17-29.

[22] N. Hirose, N. Makita, M. Kojima, T. Kamada-Nobusada, H. Sakakibara, Overexpression of a Type-A response regulator alters rice morphology and cytokinin metabolism, Plant Cell Physiol. 48 (2007) 523-539.

[23] Y. Li, D. Zhang, L. Zhang, X. Zuo, S. Fan, X. Zhang, et al., Identification and expression analysis of cytokinin responseregulator genes during floral induction in apple (Malus domestica Borkh), Plant Growth Regul. 83 (3) (2017) 455-464, https://doi.org/10.1007/s10725-017-0311-2.

[24] M. Cortizo, J.M. Álvarez, A. Rodríguez, B. Fernández, R.J. Ordás, Cloning and characterization of a type-A response regulator differentially expressed during adventitious shoot formation in Pinus pinea L, J. Plant Physiol. 167 (2010) 1023-1026.

[25] J.M. Álvarez, M. Cortizo, R.J. Ordás, Characterization of a type-A response regulator differentially expressed during adventitious caulogenesis in Pinus pinaster, J. Plant Physiol. 169 (2012) 1807-1814.

[26] G.A. Ramírez-Carvajal, A.M. Morse, J.M. Davis, Transcript profiles of the cytokinin response regulator gene family in Populus imply diverse roles in plant development, New Phytol. 177 (2008) 77-89.

[27] J. Immanen, K. Nieminen, H. Duchens Silva, F. Rodríguez Rojas, L.A. Meisel, H. Silva, et al., Characterization of cytokinin signaling and homeostasis gene families in two hardwood tree species: Populus trichocarpa and Prunus persica, BMC Genomics 14 (2013) 885-896.

[28] F. Chefdor, H. Bénédetti, C. Depierreux, F. Delmotte, D. Morabito, S. Carpin, Osmotic stress sensing in Populus: components identification of a phosphorelay system, FEBS Lett. 580 (2006) 77-81.

[29] F. Héricourt, F. Chefdor, L. Bertheau, M. Tanigawa, T. Maeda, G. Guirimand, et al., Characterization of histidine-aspartate kinase HK1 and identification of histidine phosphotransfer proteins as potential partners in a Populus multistep phosphorelay, Physiol. Planta 149 (2013) 188-199.

[30] F. Héricourt, F. Chefdor, I. Djeghdir, M. Larcher, F. Lafontaine, V. Courdavault, et al., Functional divergence of doplar histidine-aspartate kinase HK1 paralogs in response to osmotic stress, Int. J. Mol. Sci. 17 (2061) (2016), https://doi.org/10. 3390/ijms17122061.

[31] L. Bertheau, F. Chefdor, G. Guirimand, V. Courdavault, C. Depierreux, Morabito, et al., Identification of five B-type response regulators as members of a multistep phosphorelay system interacting with histidine-containing phosphotransfer partners of Populus osmosensor, BMC Plant Biol. 12 (2012) 241-255.

[32] L. Bertheau, M. Miranda, E. Foureau, L.F. Rojas Hoyos, F. Chefdor, F. Héricourt, et al., In planta validation of HK1 homodimerization and recruitment of preferential HPt downstream partners involved in poplar multistep phosphorelay systems, Plant Biosyst. Int. J. Deal. With All Asp. Plant Biol. 147 (2013) 991-995.

[33] L. Bertheau, I. Djeghdir, E. Foureau, F. Chefdor, G. Glevarec, Oudin, et al., Insights into B-type RR members as signaling partners acting downstream of HPt partners of HK1 in the osmotic stress response in Populus, Plant Physiol. Biochem. 94 (2015) $244-252$.

[34] J.D. Thompson, D.G. Higgins, T.J. Gibson, CLUSTAL W: improving the sensitivity of progressive multiple sequence alignment through sequence weighting, positionspecific gap penalties and weight matrix choice, Nucl. Acids Res. 22 (1994) 4673-4680.

[35] R.D. Gietz, R.H. Schiestl, A.R. Willems, R.A. Woods, Studies on the transformation of intact yeast cells by the LiAc/SS-DNA/PEG procedure, Yeast 11 (1995) 355-360.

[36] R. Waadt, L.K. Schmidt, M. Lohse, K. Hashimoto, R. Bock, J. Kudla, Multicolor bimolecular fluorescence complementation reveals simultaneous formation of alternative CBL/CIPK complexes in planta, Plant J. 56 (2008) 505-516.

[37] G. Guirimand, V. Courdavault, A. Lanoue, S. Mahroug, A. Guihur, N. Blanc, Strictosidine activation in Apocynaceae: towards a "nuclear time bomb"? BMC Plant Biol. 10 (2010) 182

[38] G. Guirimand, V. Burlat, A. Oudin, A. Lanoue, B. St-Pierre, V. Courdavault, Optimization of the transient transformation of Catharanthus roseus cells by particle bombardment and its application to the subcellular localization of hydroxymethylbutenyl 4-diphosphate synthase and geraniol 10-hydroxylase, Plant Cell Rep. 28 (2009) 1215-1234.

[39] A. Stavrinides, E.C. Tatsis, E. Foureau, L. Caputi, F. Kellner, V. Courdavault, S.E. O'Connor, Unlocking the diversity of alkaloids in Catharanthus roseus: nuclear localization suggests metabolic channeling in secondary metabolism, Chem. Biol. 22 (2015) 336-341.

[40] A. Caruso, D. Morabito, F. Delmotte, G. Kahlem, S. Carpin, Dehydrin induction during drought and osmotic stress in Populus, Plant Physiol. Biochem. 40 (2002) 1033-1042.

[41] B. Pils, A. Heyl, Unraveling the evolution of cytokinin signaling, Plant Physiol. 151 (2009) 782-791.

[42] N. Panchy, M. Lehti-Shiu, S.-H. Shiu, Evolution of gene duplication in plants, Plant Physiol. 171 (2016) 2294-2316.

[43] J.C. Schnable, X. Wang, J.C. Pires, M. Freeling, Escape from preferential retention following repeated whole genome duplications in plants, Front. Plant Sci. 3 (2012) 94.

[44] Y.-C. Tsai, N.R. Weir, K. Hill, W. Zhang, H.J. Kim, S.-H. Shiu, et al., Characterization of genes involved in cytokinin signaling and metabolism from rice, Plant Physiol. 158 (2012) 1666-1684.

[45] B. Zheng, Y. Deng, J. Mu, Z. Ji, T. Xiang, Q.-W. Niu, et al., Cytokinin affects circadian-clock oscillation in a phytochrome B- and Arabidopsis response regulator 4 dependent manner, Physiol. Plant. 127 (2006) 277-292.

[46] S. Nitschke, A. Cortleven, T. Schmülling, Novel stress in plants by altering the photoperiod, Trends Plant Sci. 22 (2017) 913-916. 
[47] V. Mira-Rodado, U. Sweere, C. Grefen, T. Kunkel, E. Fejes, F. Nagy, et al., Functional cross-talk between two-component and phytochrome B signal transduction in Arabidopsis, J. Exp. Bot. 58 (2007) 2595-2607.

[48] W. Chi, J. Li, B. He, X. Chai, X. Xu, X. Sun, et al., DEG9, a serine protease, modulates cytokinin and light signaling by regulating the level of ARABIDOPSIS RESPONSE REGULATOR 4, Proc. Natl. Acad. Sci. 113 (2016) E3568-E3576.

[49] H. Sakai, T. Aoyama, A. Oka, Arabidopsis ARR1 and ARR2 response regulators operate as transcriptional activators, Plant J. 24 (2000) 703-711.

[50] M.P. Williamson, The structure and function of proline-rich regions in proteins, Biochem. J. 297 (1994) 249-260.

[51] A. Geraldes, C.A. Hefer, A. Capron, N. Kolosova, F. Martinez-Nuñez, R.Y. Soolanayakanahally, et al., Recent Y chromosome divergence despite ancient origin of dioecy in poplars (Populus), Mol. Ecol. 24 (2015) 3243-3256.

[52] K. Bräutigam, R. Soolanayakanahally, M. Champigny, S. Mansfield, C. Douglas, M.M. Campbell, Q. Cronk, Sexual epigenetics: gender-specific methylation of a gene in the sex determining region of Populus balsamifera, Sci. Rep. U.K. 7 (2017) 45388, https://doi.org/10.1038/srep45388.

[53] N.V. Melnikova, E.V. Borkhert, A.V. Snezhkina, A.V. Kudryavtseva, A.A. Dmitriev, Sex-specific response to stress in Populus, Front. Plant Sci. 8 (2017) 1827, https:// doi.org/10.3389/fpls.2017.01827.

[54] F. Brignolas, C. Thierry, G. Guerrier, É. Boudouresque, Compared water deficit response of two Populus x euramericana clones, Luisa Avanzo and Dorskamp, Ann. For. Sci. 57 (2000) 261-266.

[55] X. Xu, G. Peng, C. Wu, H. Korpelainen, C. Li, Drought inhibits photosynthetic capacity more in females than in males of Populus cathayana, Tree Physiol. 28 (2008) 1751-1759.

[56] I. Efroni, E. Blum, A. Goldshmidt, Y. Eshed, A protracted and dynamic maturation schedule underlies Arabidopsis leaf development, Plant Cell 20 (2008) 2293-2306.

[57] J. Xiao, R. Jin, D. Wagner, Developmental transitions: integrating environmental cues with hormonal signaling in the chromatin landscape in plants, Genome Biol. 18 (88) (2017), https://doi.org/10.1186/s13059-017-1228-9.

[58] H. Dortay, N. Gruhn, A. Pfeifer, M. Schwerdtner, T. Schmülling, A. Heyl, Toward an interaction map of the two-component signaling pathway of Arabidopsis thaliana, J.
Proteome Res. 7 (2008) 3649-3660.

[59] P.A. Salomé, J.P.C. To, J.J. Kieber, C.R. McClung, Arabidopsis response regulators ARR3 and ARR4 play cytokinin-independent roles in the control of circadian period, Plant Cell 18 (2006) 55-69.

[60] T. Kiba, H. Yamada, T. Mizuno, Characterization of the ARR15 and ARR16 response regulators with special reference to the cytokinin signaling pathway mediated by the AHK4 histidine kinase in roots of Arabidopsis thaliana, Plant Cell Physiol. 43 (2002) 1059-1066.

[61] D.J. Lee, S. Kim, Y.-M. Ha, J. Kim, Phosphorylation of Arabidopsis response regulator 7 (ARR7) at the putative phospho-accepting site is required for ARR7 to act as a negative regulator of cytokinin signaling, Planta 227 (2008) 577-587.

[62] A. Imamura, Y. Yoshino, T. Mizuno, Cellular localization of the signaling components of Arabidopsis His-to-Asp phosphorelay, Biosci. Biotechnol. Biochem. 65 (2001) 2113-2117.

[63] V. Verma, J. Sivaraman, A.K. Srivastava, A. Sadanandom, P.P. Kumar, Destabilization of interaction between cytokinin signaling intermediates AHP1 and ARR4 modulates Arabidopsis development, New Phytol. 206 (2015) 726-737.

[64] H. Dortay, N. Mehnert, L. Bürkle, T. Schmülling, A. Heyl, Analysis of protein interactions within the cytokinin-signaling pathway of Arabidopsis thaliana, FEBS J. 273 (2006) 4631-4644.

[65] J.W. Cutcliffe, E. Hellmann, A. Heyl, A.M. Rashotte, CRFs form protein-protein interactions with each other and with members of the cytokinin signalling pathway in Arabidopsis via the CRF domain, J. Exp. Bot. 62 (2011) 4995-5002.

[66] S. Paul, H. Wildhagen, D. Janz, A. Polle, Drought effects on the tissue- and cellspecific cytokinin activity in poplar, AoB Plants 10 (2018) plx067, https://doi.org/ 10.1093/aobpla/plx067.

[67] N.Y. Kang, C. Cho, N.Y. Kim, J. Kim, Cytokinin receptor-dependent and receptorindependent pathways in the dehydration response of Arabidopsis thaliana, J. Plant Physiol. 169 (2012) 1382-1391.

[68] N.Y. Kang, C. Cho, J. Kim, Inducible expression of Arabidopsis response regulator 22 (ARR22), a type-C ARR, in transgenic Arabidopsis enhances drought and freezing tolerance, PLoS One 8 (2013) e79248, , https://doi.org/10.1371/journal. pone.0079248. 